\title{
Incidência de Tremor em Anestesia Peridural Com ou Sem Fentanil: Estudo Comparativo *
}

\section{Shivering during Epidural Anesthesia With and Without Fentanyl: Comparative Study}

Múcio Paranhos de Abreu, TSA ${ }^{1}$; João Lopes Vieira, TSA ${ }^{2}$; Marcelo Negrão Lutti, TSA ${ }^{1}$; Emily Santos Montarroyos ${ }^{3}$; Randal de Tarso Rossi ${ }^{3}$; Rodrigo Moraes ${ }^{3}$

\begin{abstract}
RESUMO
Abreu MP, Vieira JL, Lutti MN, Montarroyos ES, Rossi RT, Moraes $\mathrm{R}$ - Incidência de Tremor em Anestesia Peridural Com e Sem Fentanil: Estudo Comparativo
\end{abstract}

JUSTIFICATIVA E OBJETIVOS: A maioria dos trabalhos encontrados na literatura, relacionando a influência dos opióides administrados por via peridural com o tremor intra e pós-operatório, foram realizados com grupos de pacientes obstétricas, nas quais a resposta do centro termorregulador pode ser diferente das pacientes não grávidas. O objetivo deste trabalho foi comparar o bloqueio peridural com e sem fentanil, quanto à incidência de tremores e outras complicações no intra e pós-operatório em pacientes submetidos à cirurgia de varizes sob anestesia peridural com bupivacaína a 0,5\% com adrenalina a 1:200.000.

MÉTODO: Trinta e quatro pacientes, estado físico ASA I e II, submetidos à cirurgia para tratamento de varizes de membros inferiores, foram divididos aleatoriamente em 2 grupos $(n=17)$, e receberam midazolam $\left(0,05 \mathrm{mg} \mathrm{kg}^{-1}\right)$, por via venosa seguido de anestesia peridural lombar, utilizando-se no grupo S, $20 \mathrm{ml}$ bupivacaína a 0,5\% (com vasoconstritor) associado a $2 \mathrm{ml}$ de solução fisiológica a 0,9\% e no grupo $F, 20 \mathrm{ml}$ de bupivacaína a $0,5 \%$ (com vasoconstritor) associada ao fentanil $(100 \mu \mathrm{g})$. Foram estudados: incidência de tremor, temperatura dos pacientes, necessidade do uso de meperidina, e a incidência de náuseas e vômitos nos seguintes momentos: $M_{1}$ - admissão do paciente na sala de operação; $M_{2}$ - imediatamente antes da anestesia; $M_{3}$ - 30 minutos após o término da injeção do anestésico local; $M_{4}-60$ minutos após o término da injeção do anestésico local; $M_{5}$ - 90 minutos após o término da injeção do anestésico local; $M_{6}$ - final da anestesia; $M_{7}$ - antecedendo a alta da sala de recuperação pós-anestésica.

RESULTADOS: Quanto aos dados antropométricos, estado físico, tempo médio de duração da anestesia e cirurgia, temperatura dos pacientes e da sala de operação e incidência de náuseas e vômitos não houve diferença estatística entre os grupos. Houve diferença estatística aos 60 minutos $\left(M_{4}\right)$ e 90 minutos $\left(\mathrm{M}_{5}\right)$ após o bloqueio peridural, com maior incidência de tremor no Grupo $S$ que no Grupo F. Houve maior

\footnotetext{
* Recebido do (Received from) CET/SBA do Instituto Penido Burnier e Centro Médico de Campinas, SP

1. Instrutor do CET/SBA

2. Co-Responsável pelo CET/SBA do Instituto Penido Burnier

3. $M E_{2}$ do CET/SBA (2002)
}

Apresentado (Submitted) em 13 de março de 2003

Aceito (Accepted) para publicação em 23 de junho de 2003

Endereço para correspondência (Correspondence to)

Dr. Múcio Paranhos de Abreu

Av. Nossa Senhora de Fátima, 805/J73 Taquaral

13090-130 Campinas, SP

(C) Sociedade Brasileira de Anestesiologia, 2004 necessidade de utilização de meperidina nos pacientes submetidos ao bloqueio peridural não associado ao fentanil.

CONCLUSÕES: Nas condições deste estudo, a adição de 100 $\mu \mathrm{g}$ de fentanil ao anestésico local, por via peridural, mostrou que o opióide não tem a propriedade de abolir o tremor, mas de reduzir sua incidência e a intensidade, sem aumentar a incidência de náuseas e vômitos.

Unitermos: ANALGÉSICOS, Opióides: fentanil; COMPLICAÇÕES: hipotermia, tremores; TÉCNICAS ANESTÉSICAS, Regional: peridural

\section{SUMMARY}

Abreu MP, Vieira JL, Lutti MN, Montarroyos ES, Rossi RT, Moraes $R$ - Shivering during Epidural Anesthesia With and Without Fentanyl: Comparative Study

BACKGROUND AND OBJECTIVES: Most studies in the literature correlating epidural opioids to postoperative shivering were carried out with obstetric patients whose thermoregulation center response might be different from non-pregnant patients. Our study aimed at comparing intra and postoperative shivering and other complications of epidural block with and without fentanyl in patients submitted to varicose vein surgery under epidural anesthesia with 0.5\% bupivacaine and 1:200,000 epinephrine.

METHODS: Participated in this study 34 patients, physical status ASA I and II, submitted to lower limbs varicose vein surgery, who were randomly distributed in 2 groups $(n=17)$ and received intravenous midazolam (0.05 $\left.\mathrm{mg} \cdot \mathrm{kg}^{-1}\right)$ followed by lumbar epidural anesthesia. Group S received $20 \mathrm{~mL}$ of $0.5 \%$ bupivacaine (with epinephrine) associated to $2 \mathrm{~mL}$ of $0.9 \%$ saline solution, and Group F received $20 \mathrm{~mL}$ of $0.5 \%$ bupivacaine (with epinephrine) associated to fentanyl $(100 \mu \mathrm{g})$. Shivering, temperature, meperidine need, nausea and vomiting were evaluated in the following moments: $M_{1}$ - admission to the operating room; $M_{2}$ - immediately before anesthesia; $M_{3}$ - 30 minutes after local anesthetic injection; $M_{4}-60$ minutes after local anesthetic injection; $M_{5}-90$ minutes after local anesthetic injection; $M_{6}$ end of anesthesia; $M_{7}$ - immediately before PACU discharge.

RESULTS: There have been no statistically significant differences between groups in demographics, physical status, mean anesthesia and surgery duration, patients and operating room temperature, and the incidence of nausea and vomiting. There have been statistically significant differences in shivering occurrence at $60\left(M_{4}\right)$ and 90 minutes $\left(M_{5}\right)$ after epidural block, with higher incidence of shivering in Group $S$ as compared to Group F. A greater demand for meperidine was observed in patients submitted to epidural block without fentanyl.

CONCLUSIONS: In the conditions of our study, $100 \mu \mathrm{g}$ fentanyl associated to epidural local anesthetics did not abolish shivering but is able to decrease its incidence without increasing the incidence of nausea and vomiting.

Key Words: ANALGESICS: Opioids, fentanyl; ANESTHETIC TECHNIQUES: Regional, epidural; COMPLICATIONS: hypothermia, shivering 


\section{INTRODUÇÃO}

$A^{s}$ s complicações sistêmicas conseqüentes à anestesia peridural são bastante previsíveis e, em sua maioria, evitáveis. Os tremores que podem aparecer no intra e pós-operatório apresentam-se com alta incidência e, às vezes, de difícil controle, cuja etiologia, ainda não foi totalmente esclarecida na literatura ${ }^{1,2}$.

A diminuição da temperatura corporal, associada à vasodilatação provocada pelo bloqueio simpático, podem levar à hipotermia e conseqüentemente ao tremor.

Alguns autores têm sugerido que os tremores que ocorrem durante a anestesia peridural não resultam da hipotermia isoladamente e que a adição de opióides, como o fentanil por via peridural, parece demonstrar resultados consistentes na redução do tremor ${ }^{3-5}$. Por outro lado, a interpretação destes estudos tornam-se complicadas por diversas razões: 1) A maioria dos trabalhos publicados foram realizados com grupo de gestantes, nas quais a resposta do centro termorregulador pode ser diferente das pacientes não grávidas ${ }^{6}$; 2) Alguns estudos incluem pacientes obstétricas submetidas à analgesia de parto, juntamente com pacientes submetidas à cesariana sob anestesia peridural ${ }^{5}$; 3) Outros estudos foram realizados somente com pacientes submetidas à cesariana ${ }^{5}$.

Estudo mostrou a eficácia da meperidina, utilizando doses de 6,25 a $50 \mathrm{mg}$ por via venosa, na profilaxia e tratamento dos

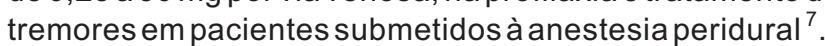

O fentanil é um opióide lipossolúvel que, associado ao anestésico local na anestesia peridural, tem a propriedade de elevar o limiar do centro termorregulador hipotalâmico para o desencadeamento do tremor ${ }^{2,8}$.

O objetivo deste estudo foi avaliar os efeitos do fentanil, administrado por via peridural juntamente com anestésico local, na prevenção de tremores no intra e pós-operatório imediato em pacientes submetidos à cirurgia de varizes de membros inferiores, sob anestesia peridural, com bupivacaína a $0,5 \%$.

\section{MÉTODO}

Após aprovação pela Comissão de Ética do Centro Médico de Campinas e consentimento formal, participaram do estudo, trinta e quatro pacientes com idades entre 21 e 62 anos, estado físico ASA I e II, submetidos à cirurgia para tratamento de varizes de membros inferiores sob anestesia peridural com bupivacaína a 0,5\% com epinefrina a 1:200.000. Os pacientes foram divididos aleatoriamente em dois grupos: grupo $F$, no qual se adicionou fentanil $(100 \mu \mathrm{g})$ por via peridural, e grupo S, sem fentanil.

Na sala de cirurgia foi realizada venóclise com cateter $20 \mathrm{G}$ em membro superior, seguida de infusão de $500 \mathrm{ml}$ de solução de Ringer com lactato, administrados antes da realização do bloqueio, aquecido previamente à temperatura de $37^{\circ} \mathrm{C}$. A reposição hídrica durante a cirurgia também foi realizada com solução de Ringer com lactato previamente aquecido a $37^{\circ} \mathrm{C}$ e infundido com equipo convencional. Foi feita monitorização não invasiva de pressão arterial, freqüência cardíaca, saturação da hemoglobina pelo oxigênio com oxímetro de pulso $\left(\mathrm{SpO}_{2}\right)$ e eletrocardiograma na derivação $D_{\|}$. A temperatura corporal dos pacientes foi medida através da aferição da temperatura timpânica com termômetro digital de ouvido, por raios infra-vermelhos, de uso intermitente e graduado para escala em graus Celsius $\left({ }^{\circ} \mathrm{C}\right)$. A temperatura da sala cirúrgica foi observada continuamente por termômetro digital ambiental.

Todos os pacientes receberam midazolam $\left(0,05 \mathrm{mg} \cdot \mathrm{kg}^{-1}\right)$ por via venosa, imediatamente antes da anestesia peridural. A punção peridural foi realizada com o paciente na posição sentada, utilizando-se agulha $17 \mathrm{G}$ tipo Weiss, com a identificação do espaço peridural lombar $\left(L_{2}-L_{3}\right.$ ou $\left.L_{3}-L_{4}\right)$, através da técnica de perda da resistência à injeção de ar. Após realização de aspiração e dose-teste com $3 \mathrm{ml}$ de lidocaína a $2 \%$ com epinefrina 1:200.000, utilizaram-se no grupo S, $20 \mathrm{ml}$ de bupivacaína a 0,5\% (com vasoconstritor) associados a $2 \mathrm{ml}$ de solução fisiológica a $0,9 \%$ e no grupo $F, 20 \mathrm{ml}$ de bupivacaína a $0,5 \%$ (com vasoconstritor) associados a $2 \mathrm{ml}$ de fentanil $(100 \mu \mathrm{g})$.

Em todos os pacientes administrou-se rotineiramente oxigênio $\left(2\right.$ L. $\left.\mathrm{min}^{-1}\right)$ através de cateter nasal.

Registraram-se os valores obtidos para os dados hemodinâmicos e temperatura do paciente, temperatura do ambiente cirúrgico e a presença de tremores, em sete momentos :

$M_{1}$ - na admissão do paciente na sala de operação;

$\mathrm{M}_{2}$ - imediatamente antes da anestesia peridural;

$\mathrm{M}_{3}$ - 30 minutos após o término da injeção do anestésico local (AL);

$M_{4}-60$ minutos após o término da injeção do $A L$;

$M_{5}$ - 90 minutos após o término da injeção do $A L$;

$\mathrm{M}_{6}$ - término da anestesia;

$\mathrm{M}_{7}$ - antecedendo a alta da sala de recuperação pós-anestésica.

Aavaliação da presença de tremores foi realizada pelo anestesiologista e classificados como 0 = ausência de tremor; $1=$ fasciculação na musculatura masseteriana; 2= fasciculações musculares na face, pescoço e extremidades superiores e $3=$ abalos musculares. O tratamento com meperidina, na dose de 5 a $50 \mathrm{mg}$ por via venosa, ficaria reservado aos pacientes que apresentassem tremor classe 2 ou 3 . Adose inicial foi padronizada em $5 \mathrm{mg}$, sendo que incrementos de $5 \mathrm{mg}$ seriam administrados até a abolição do tremor, não ultrapassando $50 \mathrm{mg}$. Os efeitos adversos, como náuseas e vômitos, foram registrados. Os dados foram comparados entre os dois grupos.

Para a análise das variáveis idade, peso e altura utilizou-se o teste $t$ de Student. Para as variáveis sexo e estado físico foi utilizado o teste do Qui-quadrado. Para comparar o tempo para aparecimento e incidência dos tremores, bem como a necessidade do uso de meperidina nos dois grupos, utilizou-se a prova não paramétrica de Mann-Whitney, para duas amostras independentes. Foi estabelecido o valor de $p<0,05$ como significante. 


\section{RESULTADOS}

Quanto aos dados antropométricos, estado físico, tempo médio de duração da anestesia e cirurgia, temperatura do paciente e da sala de operações, bem como a incidência de náuseas e vômitos, não houve diferença estatística entre os grupos (Tabelas I, II, III e IV). Houve diferença estatística aos 60 minutos $\left(\mathrm{M}_{4}\right)$ e 90 minutos $\left(\mathrm{M}_{5}\right)$ após o bloqueio peridural, com maior incidência de tremor no Grupo $S$ do que no Grupo F (Tabela V). A intensidade dos tremores também foi maior no Grupo $S$ que no Grupo F nos momentos $\mathrm{M}_{4}$ e $\mathrm{M}_{5}$ (Tabela VI).

Houve maior necessidade de utilização de meperidina nos pacientes submetidos ao bloqueio peridural não associado ao fentanil (Grupo S). A dose média de meperidina utilizada no grupo $S$ foi de $26,67 \pm 11,69$. No grupo F, o emprego de meperidina $(30 \mathrm{mg}$ ) foi necessário em apenas um caso (Tabela VII).
Tabela IV - Náuseas e Vômitos

\begin{tabular}{lcccc}
\hline & \multicolumn{2}{c}{ Náuseas } & \multicolumn{2}{c}{ Vômitos } \\
\hline Grupos & N $^{\circ}$ de pacientes & $\%$ & $N^{\circ}$ de pacientes & $\%$ \\
\hline GS $(n=17)$ & 1 & 5,9 & 1 & 5,9 \\
GF $(n=17)$ & 3 & 17,65 & 0 & 0 \\
\hline
\end{tabular}

Não houve diferença estatística entre os grupos

Tabela V - Incidência de Tremores nos Momentos de $\mathrm{M}_{2}$ a $\mathrm{M}_{7}$

\begin{tabular}{lcccccc} 
Grupos & $M_{2}$ & $M_{3}$ & $M_{4}^{*}$ & $M_{5}^{*}$ & $M_{6}$ & $M_{7}$ \\
\hline GS $(n=17)$ & 0 & 1 & $10^{*}$ & $8^{*}$ & 6 & 5 \\
GF $(n=17)$ & 0 & 5 & $7^{*}$ & $5^{*}$ & 7 & 5 \\
\hline
\end{tabular}

* Diferença estatística nos momentos $\mathrm{M}_{4}(60 \mathrm{~min})$ e $\mathrm{M}_{5}(90 \mathrm{~min})$

Tabela VI- Número de Pacientes e Dose de Meperidina Utilizada

\begin{tabular}{lcc}
\hline Grupos & $N^{\circ}$ de pacientes & Dose de meperidina $(\mathrm{mg})$ \\
\hline GS $(n=17)$ & 6 & $26,67 \pm 11,69$ \\
GF $(n=17)$ & 1 & 30 \\
\hline
\end{tabular}

Diferença estatística entre os grupos, quanto ao número de pacientes em que se utilizou meperidina

Tabela I - Dados Antropométricos de Ambos os Grupos, Sexo e Estado Físico (ASA)

\begin{tabular}{|c|c|c|c|c|c|c|c|}
\hline \multirow[t]{2}{*}{ Grupos } & \multirow{2}{*}{$\begin{array}{c}\text { Idade * } \\
\text { (anos) }\end{array}$} & \multirow{2}{*}{$\begin{array}{l}\text { Peso* } \\
\text { kg) }\end{array}$} & \multirow{2}{*}{$\begin{array}{c}\text { Altura * } \\
\text { cm) }\end{array}$} & \multicolumn{2}{|c|}{ Sexo } & \multicolumn{2}{|c|}{ Estado Físico } \\
\hline & & & & $\mathrm{F}$ & $M$ & ASA I & ASA II \\
\hline GS $(n=17)$ & $39,12 \pm 9,47$ & $71,70 \pm 21,47$ & $167,29 \pm 8,84$ & 15 & 2 & 13 & 4 \\
\hline $\mathrm{GF}(n=17)$ & $40,05 \pm 9,15$ & $69,55 \pm 16,61$ & $163,18 \pm 6,66$ & 13 & 4 & 15 & 2 \\
\hline
\end{tabular}

Não houve diferença estatística entre os grupos

* Valores expressos pela Média \pm DP

Tabela II - Temperatura da Sala de Operações nos Momentos de $M_{2}$ a $M_{7}$ (Média \pm DP)

\begin{tabular}{lcccccc}
\hline Grupos & $M_{2}$ & $M_{3}$ & $M_{4}$ & $M_{5}$ & $M_{6}$ & $M_{7}$ \\
\hline GS $(n=17)$ & $20,58 \pm 1,28$ & $20,28 \pm 1,04$ & $20,44 \pm 0,84$ & $20,55 \pm 0,82$ & $20,81 \pm 0,78$ & $23,07 \pm 0,96$ \\
GF $(n=17)$ & $20,46 \pm 1,71$ & $20,64 \pm 1,58$ & $20,51 \pm 1,42$ & $20,68 \pm 1,33$ & $20,99 \pm 1,46$ & $23,32 \pm 1,18$ \\
\hline
\end{tabular}

Não houve diferença estatística entre os grupos

Tabela III - Temperatura dos Pacientes nos Momentos de $M_{1}$ a $M_{7}$ (Média \pm DP)

\begin{tabular}{|c|c|c|c|c|c|c|c|}
\hline Grupos & $\mathrm{M}_{1}$ & $\mathrm{M}_{2}$ & $\mathrm{M}_{3}$ & $\mathrm{M}_{4}$ & $\mathrm{M}_{5}$ & $M_{6}$ & $\mathrm{M}_{7}$ \\
\hline GS $n=17)$ & $35,94 \pm 0,54$ & $35,84 \pm 0,45$ & $35,09 \pm 0,38$ & $34,57 \pm 0,40$ & $34,58 \pm 0,37$ & $34,48 \pm 0,55$ & $34,82 \pm 0,89$ \\
\hline GF $(n=17)$ & $36,11 \pm 0,56$ & $35,78 \pm 0,58$ & $35,04 \pm 0,57$ & $34,71 \pm 0,59$ & $34,65 \pm 0,64$ & $34,76 \pm 0,66$ & $35,31 \pm 1,36$ \\
\hline
\end{tabular}

Não houve diferença estatística entre os grupos 
Tabela VII - Número de Pacientes com Diferentes Intensidades de Tremores nos Momentos de $\mathrm{M}_{2}$ a $\mathrm{M}_{7}$

\begin{tabular}{|c|c|c|c|c|c|c|c|}
\hline $\begin{array}{l}\text { Classificação } \\
\text { dos Tremores }\end{array}$ & $\mathrm{M}_{2}$ & $M_{3}$ & $\mathrm{M}_{4}{ }^{*}$ & $\mathrm{M}_{5}{ }^{*}$ & $M_{6}$ & $\mathrm{M}_{7}$ & $\begin{array}{l}N^{\circ} \text { Pacientes } \\
\text { que Receberam } \\
\text { Meperidina }\end{array}$ \\
\hline \multicolumn{8}{|l|}{$G S(n=17)$} \\
\hline 0 & 17 & 16 & 7 & 9 & 11 & 12 & 0 \\
\hline 1 & 0 & 0 & 6 & 7 & 6 & 5 & 0 \\
\hline 2 & 0 & 0 & 4 & 1 & 0 & 0 & 5 \\
\hline 3 & 0 & 1 & 0 & 0 & 0 & 0 & 1 \\
\hline \multicolumn{8}{|l|}{ GF $(n=17)$} \\
\hline 0 & 17 & 12 & 10 & 12 & 10 & 12 & 0 \\
\hline 1 & 0 & 4 & 7 & 5 & 7 & 5 & 0 \\
\hline 2 & 0 & 1 & 0 & 0 & 0 & 0 & 1 \\
\hline 3 & 0 & 0 & 0 & 0 & 0 & 0 & 0 \\
\hline
\end{tabular}

* Diferença estatística nos momentos $\mathrm{M}_{4}$ e $\mathrm{M}_{5}$

\section{DISCUSSÃo}

Os tremores que podem ocorrer durante a anestesia peridural são considerados como uma resposta do centro termorregulador hipotalâmico e, apesar de ainda não estar bem claro na literatura, dentre outras causas, devem-se principalmente à hipotermia que pode advir da injeção peridural de anestésico local e, resultantes em parte, da redistribuição de calor corporal da região central para região periférica ${ }^{3,9-15}$. A ocorrência de hipotermia leve ou moderada $\left(35,9\right.$ a $\left.34^{\circ} \mathrm{C}\right)$ durante anestesia geral ou regional desencadeia no organismo a ativação de mecanismos termorreguladores responsáveis pela diminuição da perda da temperatura e aumento na produção de calor: tremores musculares, hiperatividade dos centros simpáticos provocando vasoconstrição da região acima do bloqueio simpático, aumento das reações enzimáticas pelas catecolaminas, abolição da sudação, entre outros.

Outros fatores que contribuem para a diminuição da temperatura corporal envolvem a baixa temperatura da sala cirúrgica (abaixo de $21^{\circ} \mathrm{C}$ ) com proteção insuficiente contra perda de calor; anti-sepsia da pele com soluções alcoólicas frias; reposição de volume com soluções à temperatura ambiente; administração de drogas anestésicas que deprimem a atividade metabólica produtora de calor, levando à vasodilatação da pele e conseqüente aumento da perda de calor.

Aetiologia dos tremores que freqüentemente ocorrem no período intra e pós-anestésico podem ainda estar relacionada com a inibição dos reflexos medulares, bloqueio regional da atividade simpática levando à vasodilatação periférica e perda de calor, liberação de substâncias pirogênicas, entre outras causas ${ }^{13}$

As conseqüências destes tremores são proporcionais à intensidade dos mesmos e podem aumentar o consumo de oxigênio em $200 \%$ a $800 \%$ acima dos valores basais ${ }^{13}$. Nos pacientes com reserva cardíaca diminuída, este aumento no consumo de oxigênio poderá culminar com a redução na oferta tecidual de oxigênio, contribuindo para o aparecimen- to de isquemia do miocárdio e aumento da incidência de disritmias ventriculares no pós-operatório ${ }^{16,17}$.

Dentre os efeitos colaterais associados com a anestesia peridural, como náuseas, vômitos, cefaléias e outros, o tremor foi mencionado como uma experiência desagradável e muito desconfortável para os pacientes ${ }^{18,19}$.

A profilaxia dos tremores durante anestesia peridural inclui, entre outras, algumas medidas, como:

- Aquecer os fluidos infundidos a $37^{\circ} \mathrm{C}{ }^{1,21}$;

- Manutenção da temperatura da sala de cirurgia entre 21 ${ }^{\circ} \mathrm{C}$ e $24{ }^{\circ} \mathrm{C}{ }^{18}$;

- Uso de colchões e mantas térmicas;

- Emissão de calor irradiante, podendo aumentar a temperatura sangüínea central em até $17,7 \mathrm{kcal} . \mathrm{h}^{-1}{ }^{23}$.

Dependendo do tipo de cirurgia, da duração do procedimento, exposição de vísceras etc., essas medidas isoladamente podem não prevenir a hipotermia e os tremores.

Foi descrito que os tremores podem ser abolidos através da administração de opióides por via venosa, levando a um aumento da tolerância à hipotermia, devido à diminuição da temperatura central para desencadear o tremor ${ }^{2,3,22,23}$. A administração de opióides por via peridural também pode apresentar a propriedade de abolir os tremores, embora os mecanismos de ação desses opióides, influenciando no limiar do tremor, ainda não estejam bem estabelecidos ${ }^{2,6}$. Sabe-se que o fentanil, quando administrado por via peridural, tem sua fração não ionizada rapidamente transferida através da dura-máter para o líquor e, deste, para a medula espinhal. Ele chega à medula através da absorção pelas artérias radiculares. Ocorre também rápida absorção sistêmica, originando concentrações plasmáticas significativas de fentanil, resultando em ação analgésica sistêmica e medu$\operatorname{lar}^{2,25,26}$.

A maneira como o fentanil administrado por via peridural chega ao centro termorregulador, influenciando no limiar do tremor, ainda não é totalmente conhecida. Devido à sua lipossolubili- 
dade, pequenas concentrações de fentanil alcançam o cérebro através do líquido cerebroespinhal; portanto, ainda não se conhece o mecanismo pelo qual o fentanil atinge o hipotálamo em concentração suficiente para interferir no limiar de tremor ${ }^{2,25}$. Acredita-se que estas concentrações são alcançadas através da absorção sistêmica do fentanil peridural ${ }^{26}$.

Nesse estudo, a ocorrência de tremores foi maior aos 60 e 90 minutos após a injeção peridural no grupo em que não se utilizou fentanil, sendo o resultado estatisticamente significativo em relação ao grupo em que se associou fentanil. No entanto, mostra também que o emprego de fentanil não aboliu a presença de tremores. Quanto à intensidade dos mesmos, ela foi maior no grupo sem fentanil, resultando em maior número de pacientes (6) em que foi necessário o emprego de meperidina, por via venosa. No grupo em que foi utilizado fentanil, apenas em um caso foi necessário o emprego de meperidina. Utilizando doses fracionadas de meperidina, em nenhum caso a dose chegou a $50 \mathrm{mg}$ (Grupo $\mathrm{S}=26,67 \pm$ 11,89 e Grupo $\mathrm{F}=30 \mathrm{mg}$ ).

As desvantagens do uso de opióides por via peridural estão relacionadas com os seus efeitos colaterais: prurido, náuseas e vômitos, retenção urinária, sedação e depressão respiratória, que, embora importantes, são pouco freqüentes, quando for usado em baixas doses.

Neste estudo não houve diferença estatística quanto à incidência de náuseas e vômitos entre os grupos com ou sem fentanil.

Podemos concluir que nas condições deste estudo, a adição de $100 \mu \mathrm{g}$ de fentanil ao anestésico local, por via peridural, mostrou que o opióide não tem a propriedade de abolir o tremor, mas de reduzir sua incidência e a intensidade, sem aumentar a incidência de náuseas e vômitos.

\section{Shivering during Epidural Anesthesia With and Without Fentanyl: Comparative Study}

Múcio Paranhos de Abreu, TSA, M.D.; João Lopes Vieira, TSA, M.D.; Marcelo Negrão Lutti, TSA, M.D.; Emily Santos Montarroyos, M.D.; Randal de Tarso Rossi, M.D.; Rodrigo Moraes, M.D.

\section{INTRODUCTION}

Systemic epidural anesthesia complications are predictable and mostly avoidable. There is a high incidence of intra and postoperative shivering, which is sometimes difficult to control, the etiology of which has not been totally explained by the literature ${ }^{1,2}$

Decreased body temperature induced by the sympathetic block-induced vasodilation may lead to hypothermia and, consequently, to shivering.

Some authors have suggested that shivering during epidural anesthesia does not result from hypothermia alone and that the association of epidural opioids, such as fentanyl, seems to have consistent results in decreasing shivering ${ }^{3-5}$. On the other hand, interpreting those studies is cumbersome for several reasons: 1) most studies were carried out with pregnant patients whose thermoregulation center response might be different from non-pregnant patients ${ }^{6}$; 2) some studies have included obstetric patients submitted to labor analgesia together with patients submitted to $\mathrm{C}$-section under epidural anesthesia ${ }^{5}$; 3 ) other studies were carried out just with patients submitted to $\mathrm{C}$-section ${ }^{5}$.

A study has shown the efficacy of 6.25 to $50 \mathrm{mg}$ intravenous meperidine to prevent and control shivering in patients submitted to epidural anesthesia ${ }^{7}$.

Fentanyl is a liposoluble opioid which, associated to local anesthetics in epidural anesthesia, is able to increase hypothalamic thermoregulation center threshold for triggering shivering ${ }^{2,8}$.

This study aimed at evaluating the effects of epidural fentanyl associated to local anesthetics in preventing intra and immediate postoperative shivering in patients submitted to lower limb varicose vein surgery under epidural anesthesia with $0.5 \%$ bupivacaine plus epinephrine 1:200,000.

\section{METHODS}

After the Centro Médico de Campinas Ethics Committee approval and their formal consent, participated in this study 34 patients aged 21 to 62 years, physical status ASA I and II, submitted to lower limb varicose vein surgery under epidural anesthesia with $0.5 \%$ bupivacaine and 1:200,000 epinephrine. Patients were randomly distributed in two groups: Group F, with epidural fentanyl $(100 \mu \mathrm{g})$ and Group S, without fentanyl. Venoclysis was performed with $20 \mathrm{G}$ catheter in the operating room in an upper limb followed by $500 \mathrm{ml}$ previously warmed to $37^{\circ} \mathrm{C}$ lactated Ringer's solution infusion before blockade. The same warmed solution was used for intraoperative hydration and was infused with conventional device. Monitoring parameters were: noninvasive blood pressure, heart rate, oxygen hemoglobin saturation with pulse oximetry $\left(\mathrm{SpO}_{2}\right)$ and $E C G$ at $D_{\|}$lead. Body temperature was measured through tympanic temperature with digital infrared ear thermometer of intermittent use and graduated in degrees Celsius $\left({ }^{\circ} \mathrm{C}\right)$. Operating room temperature was continuously monitores with an environmental digital thermometer.

All patients received intravenous midazolam $\left(0.05 \mathrm{mg} \cdot \mathrm{kg}^{-1}\right)$ immediately before epidural anesthesia. Epidural puncture was performed with patients in the sitting position, with $17 \mathrm{G}$ Weiss needle and identification of lumbar epidural space $\left(L_{2}-L_{3}\right.$ or $\left.L_{3}-L_{4}\right)$, through the loss of resistance to air technique. After aspiration and test dose with $3 \mathrm{ml}$ of $2 \%$ lidocaine with $1: 200,000$ epinephrine, Group S received $20 \mathrm{~mL}$ of $0.5 \%$ bupivacaine (with epinephrine) associated to $2 \mathrm{~mL}$ of $0.9 \%$ saline solution, and Group F received $20 \mathrm{~mL}$ of $0.5 \%$ bupivacaine (with vasoconstrictor) associated to $2 \mathrm{~mL}$ fentanyl $(100 \mu \mathrm{g}$ ). All patients received routinely oxygen $\left(2 \mathrm{~L}\right.$. $\left.\mathrm{min}^{-1}\right)$ through a nasal catheter. Hemodynamic data, patients and operating room temperature and the presence of shivering were recorded in seven moments: 
$M_{1}$ - at operating room admission;

$\mathrm{M}_{2}$ - immediately before epidural anesthesia;

$\mathrm{M}_{3}-30$ minutes after local anesthetic injection (LA);

$\mathrm{M}_{4}-60$ minutes after LA injection;

$M_{5}$ - 90 minutes after LA injection;

$\mathrm{M}_{6}$ - end of anesthesia;

$\mathrm{M}_{7}$ - immediately before PACU discharge.

The incidence of shivering was evaluated by the anesthesiologist and was classified as $0=$ no shivering; 1 = masseter muscle fasciculation; 2 = face, neck and upper extremities muscles fasciculation and $3=$ muscle shakes. Intravenous 5 to $50 \mathrm{mg}$ meperidine was used in patients with shivering class 2 or 3 . Initial dose was standardized in $5 \mathrm{mg}$ and $5 \mathrm{mg}$ increments would be added until shivering disappearance, however not beyond $50 \mathrm{mg}$. Adverse effects, such as nausea and vomiting were recorded and data were compared between groups.

Student's $t$ test was used to compare age, weight and height. Chi-square test was used to compare gender and physical status. Non-parametric Mann-Whitney test for two independent samples was used to compare onset and incidence of shivering as well as meperidine consumption for both groups, considering significant $p<0.05$.

\section{RESULTS}

No statistically significant differences were observed between groups in demographics, physical status, mean anesthesia and surgery duration, patients and operating room temperature, and the incidence of nausea and vomiting ( $\mathrm{Ta}$ bles I, II, III and IV). A significant difference on shivering occurrence at $60\left(\mathrm{M}_{4}\right)$ and 90 minutes $\left(\mathrm{M}_{5}\right)$ after epidural block was observed, with a higher incidence of shivering in Group S as compared to Group F (Table V). Shivering intensity was also higher in Group $S$ as compared to Group $F$ in moments $\mathrm{M}_{4}$ and $\mathrm{M}_{5}$ (Table $\mathrm{VI}$ ).

Table IV - Nausea and Vomiting

\begin{tabular}{lcccc}
\hline & Nausea & \multicolumn{2}{c}{ Vomiting } \\
\hline Groups & Number of Patients & $\%$ & Number of Patients & $\%$ \\
\hline GS $(n=17)$ & 1 & 5.9 & 1 & 5.9 \\
GF $(n=17)$ & 3 & 17.65 & 0 & 0 \\
\hline
\end{tabular}

There were no statistical differences between groups

Table $V$ - Incidence of Shivering in Moments $M_{2}$ to $M_{7}$

\begin{tabular}{lcccccc}
\hline Groups & $M_{2}$ & $M_{3}$ & $M_{4}{ }^{*}$ & $M_{5}{ }^{*}$ & $M_{6}$ & $M_{7}$ \\
\hline GS $(n=17)$ & 0 & 1 & $10^{*}$ & $8^{*}$ & 6 & 5 \\
GF $(n=17)$ & 0 & 5 & $7^{*}$ & $5^{*}$ & 7 & 5 \\
\hline
\end{tabular}

* Statistical Difference in moments $M_{4}(60 \mathrm{~min})$ and $M_{5}(90 \mathrm{~min})$

Table VI - Number of Patients and Meperidine Dose

\begin{tabular}{lcc}
\hline Groups & Number of Patients & Meperidine Dose $(\mathrm{mg})$ \\
\hline GS $(n=17)$ & 6 & $26.67 \pm 11.69$ \\
GF $(n=17)$ & 1 & 30 \\
\hline
\end{tabular}

Statistical difference between groups in the number of patients needing meperidine

Patients submitted to epidural block without fentanyl (Group S) needed more meperidine. Mean meperidine dose for Group S was $26.67 \pm 11.69$. Meperidine was only needed for one Group F patient (Table VII).

Table I - Demographics Data, Gender and Physical Status (ASA) for Both Groups

\begin{tabular}{|c|c|c|c|c|c|c|c|}
\hline \multirow[t]{2}{*}{ Groups } & \multirow{2}{*}{$\begin{array}{l}\text { Age * } \\
\text { (years) }\end{array}$} & \multirow{2}{*}{$\begin{array}{l}\text { Weight* } \\
(\mathrm{kg})\end{array}$} & \multirow{2}{*}{$\begin{array}{l}\text { Height * } \\
(\mathrm{cm})\end{array}$} & \multicolumn{2}{|c|}{ Gender } & \multicolumn{2}{|c|}{ Physical Status } \\
\hline & & & & $\mathrm{F}$ & M & ASA I & ASA II \\
\hline$G S(n=17)$ & $39.12 \pm 9.47$ & $71.70 \pm 21.47$ & $167.29 \pm 8.84$ & 15 & 2 & 13 & 4 \\
\hline$G F(n=17)$ & $40.05 \pm 9.15$ & $69.55 \pm 16.61$ & $163.18 \pm 6.66$ & 13 & 4 & 15 & 2 \\
\hline
\end{tabular}

There were no statistical differences between groups

*Values expressed in Mean \pm SD

Table II - Operating Room Temperature in Moments $M_{2}$ to $M_{7}$ (Mean $\pm S D$ )

\begin{tabular}{lcccccc}
\hline Groups & $M_{2}$ & $M_{3}$ & $M_{4}$ & $M_{5}$ & $M_{6}$ & $M_{7}$ \\
\hline GS $(n=17)$ & $20.58 \pm 1.28$ & $20.28 \pm 1.04$ & $20.44 \pm 0.84$ & $20.55 \pm 0.82$ & $20.81 \pm 0.78$ & $23.07 \pm 0.96$ \\
GF $(n=17)$ & $20.46 \pm 1.71$ & $20.64 \pm 1.58$ & $20.51 \pm 1.42$ & $20.68 \pm 1.33$ & $20.99 \pm 1.46$ & $23.32 \pm 1.18$ \\
\hline
\end{tabular}

There were no statistical differences between groups

Table III - Patients Temperature in Moments $M_{1}$ to $M_{7}$ (Mean $\pm S D$ )

\begin{tabular}{|c|c|c|c|c|c|c|c|}
\hline Groups & $\mathrm{M}_{1}$ & $\mathrm{M}_{2}$ & $M_{3}$ & $\mathrm{M}_{4}$ & $\mathrm{M}_{5}$ & $\mathrm{M}_{6}$ & $\mathrm{M}_{7}$ \\
\hline GS n=17) & $35.94 \pm 0.54$ & $35.84 \pm 0.45$ & $35.09 \pm 0.38$ & $34.57 \pm 0.40$ & $34.58 \pm 0.37$ & $34.48 \pm 0.55$ & $34.82 \pm 0.89$ \\
\hline GF $(n=17)$ & $36.11 \pm 0.56$ & $35.78 \pm 0.58$ & $35.04 \pm 0.57$ & $34.71 \pm 0.59$ & $34.65 \pm 0.64$ & $34.76 \pm 0.66$ & $35.31 \pm 1.36$ \\
\hline
\end{tabular}

There were no statistical differences between groups 
Table VII - Number of Patients with Different Shivering Intensities in Moments $M_{2}$ to $M_{7}$

\begin{tabular}{|c|c|c|c|c|c|c|c|}
\hline $\begin{array}{l}\text { Shivering } \\
\text { Classification }\end{array}$ & $\mathrm{M}_{2}$ & $\mathrm{M}_{3}$ & $\mathrm{M}_{4}{ }^{*}$ & $\mathrm{M}_{5}{ }^{*}$ & $M_{6}$ & $\mathrm{M}_{7}$ & $\begin{array}{l}\text { Number of Patients } \\
\text { Receiving Meperidine }\end{array}$ \\
\hline \multicolumn{8}{|l|}{ GS $(n=17)$} \\
\hline 0 & 17 & 16 & 7 & 9 & 11 & 12 & 0 \\
\hline 1 & 0 & 0 & 6 & 7 & 6 & 5 & 0 \\
\hline 2 & 0 & 0 & 4 & 1 & 0 & 0 & 5 \\
\hline 3 & 0 & 1 & 0 & 0 & 0 & 0 & 1 \\
\hline \multicolumn{8}{|l|}{ GF $(n=17)$} \\
\hline 0 & 17 & 12 & 10 & 12 & 10 & 12 & 0 \\
\hline 1 & 0 & 4 & 7 & 5 & 7 & 5 & 0 \\
\hline 2 & 0 & 1 & 0 & 0 & 0 & 0 & 1 \\
\hline 3 & 0 & 0 & 0 & 0 & 0 & 0 & 0 \\
\hline
\end{tabular}

* Statistical difference in moments $\mathrm{M}_{4}$ and $\mathrm{M}_{5}$

\section{DISCUSSION}

Epidural anesthesia shivering is considered a response of hypothalamic thermoregulation center and, although not being clear in the literature, seems to be due to hypothermia caused by local epidural anesthetic injection and partially resulting from the redistribution of body warmth from the central to the peripheral region ${ }^{3,9-15}$, among other causes. Mild or moderate hypothermia $\left(35.9\right.$ to $34^{\circ} \mathrm{C}$ ) during general or regional anesthesia triggers the activation of thermoregulatory mechanisms responsible for decreasing temperature loss and increasing heat generation: muscle shivering, sympathetic centers hyperactivity promoting vasoconstriction in the area above sympathetic block, increase in enzyme reactions by catecholamines and elimination of sweating, among others.

Other factors contributing to body temperature decrease involve low operating room temperature (below $21^{\circ} \mathrm{C}$ ) with insufficient protection against heat loss; skin cleaning with cold alcoholic solutions; volume replacement with room temperature solutions; administration of anesthetic drugs depressing metabolic heat-producing activity, leading to skin vasodilation and consequent increase in heat loss.

Intra and post-anesthetic shivering etiology may too be related to the inhibition of medullary reflexes, regional sympathetic activity block leading to peripheral vasodilation and heat loss and pyrogenic substances release, among other causes ${ }^{13}$.

Shivering consequences are a function of its intensity and may increase oxygen consumption in $200 \%$ to $800 \%$ above baseline values ${ }^{13}$. In patients with decreased cardiac reserves, this oxygen consumption increase may peak with the decrease in tissue oxygen supply, contributing for increased incidence of postoperative ventricular arrhythmias ${ }^{16,17}$.

Among epidural anesthesia-related side-effects, such as nausea, vomiting and headache among others, shivering has been mentioned as a disagreeable and very uncomfortable experience ${ }^{18,19}$.

Shivering prevention during epidural anesthesia includes some measures such as:

- Warming infused fluids to $37^{\circ} \mathrm{C}{ }^{1,21}$;

- Maintaining operating room temperature between 21 and $24{ }^{\circ} \mathrm{C}^{18}$;

- Using thermal mattresses and blankets;

- Irradiating heat emission to increase central blood temperature in up to $17.7 \mathrm{kcal} . \mathrm{h}^{-1} 23$.

Depending on surgery type and duration, visceral exposure, etc., these measures alone may not prevent hypothermia and shivering.

It has been described that shivering may be abolished by intravenous opioids, leading to increased tolerance to hypothermia due to decrease in central temperature needed to trigger shivering $2,3,22,23$.

Epidural opioids may also abolish shivering although their mechanism of action on shivering threshold is still not well defined $^{2,6}$.

It is known that epidural fentanyl has its non-ionized fraction rapidly transferred through the dura to CSF and from it to spinal cord. It reaches spinal cord through the absorption by radicular arteries. There is also a fast systemic absorption originating significant plasma fentanyl concentrations resulting in systemic and medullary analgesic action ${ }^{2,25,26}$. How epidural fentanyl reaches thermoregulatory center and influences shivering threshold is still not totally known. Due to its liposolubility, low fentanyl concentrations reach the brain through CSF; so, the mechanism by which fentanyl reaches hypothalamus in concentrations enough to interfere with shivering threshold is still not known ${ }^{2,25}$. It is believed that such concentrations are reached through systemic epidural fentanyl absorption ${ }^{26}$. 
In our study, the incidence of shivering was significantly higher at 60 and 90 minutes after epidural injection in the group not receiving fentanyl, as compared to the group receiving fentanyl, although fentanyl has not abolished shivering. Shivering intensity was also higher in the group without fentanyl and resulting in a greater number of patients (6) needing intravenous meperidine. Only one patient needed meperidine in the group receiving fentanyl. With fractioned meperidine doses, no patient has reached $50 \mathrm{mg}$ (Group $\mathrm{S}=$ $26.67 \pm 11.89$ and Group F = $30 \mathrm{mg}$ ).

Disadvantages of epidural opioids are their side-effects: pruritus, nausea and vomiting, urinary retention, sedation and respiratory depression which, although important, are not frequent when opioids are used in low doses.

No significant differences in the incidence of nausea and vomiting were observed in our study.

One may conclude that, in the conditions of our study, the association of $100 \mu \mathrm{g}$ fentanyl to epidural local anesthetics is unable to abolish shivering but is able to decrease its incidence and intensity without increasing the incidence of nausea and vomiting.

\section{REFERÊNCIAS - REFERENCES}

01. Carvalho MJ, Carvalho JCA, Castellana MEB et al - Tremores durante anestesia peridural: influência da temperatura do anestésico local e dos líquidos infundidos. Rev Bras Anestesiol, 1989;39:187-190.

02. Wheelahan JM, Leslie K, Silbert BS - Epidural fentanyl reduces the shivering threshold during epidural lidocaine anesthesia. Anesth Analg, 1998;87:587-590.

03. Matthews N, Corser G - Epidural fentanyl for shaking in obstetrics. Anaesthesia, 1988;43:783-785.

04. Ponte J, Sessler DI - Extradurals and shivering: effects of cold and warm extradural saline injections in volunteers. $\mathrm{Br} J$ Anaesth, 1990;64:731-733.

05. Liu WH, Luxton MC - The effect of prophylactic fentanyl on shivering in elective caesarian section under epidural analgesia.

06. Rolbin SH - Editorial review of four papers on shivering. Obstetric Anesth Digest, 1987;7:64.

07. Imbelloni LE - Meperidina para controle do tremor transoperatório durante cesariana sob anestesia peridural. Rev Bras Anestesiol, 1989;39:343-347

08. Shehabi Y, Gatt S, Buckman T et al - Effect of adrenaline, fentanyl and warming of injectate on shivering following extradural analgesia in labour. Anaesth Intensive Care, 1990;18:31-37.

09. Cousins MJ, Veering BT - Epidural Neural Blockade, em: Cousins MJ, Bridenbaugh - Neural Blockade in Clinical Anesthesia and Management of Pain, $3^{\text {rd }}$ Ed, Philadelphia, Lippincott-Raven Publishers, 1998;8:243-320.

10. Glosten B, Hynson J, Sessler DI et al - Preanesthetic skin-surface warming reduces redistribution hypothermia caused by epidural block. Anesth Analg, 1993;77:488-493.

11. Hynson JM, Sessler DI, Glosten B et al - Thermal balance and tremor patterns during epidural anesthesia. Anesthesiology,

12. Sessler DI, Ponte J - Shivering during epidural anesthesia. Anesthesiology, 1990;72:816-821. Anaesthesia, 1991:46:344-348. 1991;74:680-690.

13. Silva ED, Quinto D - Controle da Hipotermia Acidental, em: Yamashita AM - Atualização em Anestesiologia, São Paulo, Office Editora, 1999:111-129.

14. Sessler DI, McGuire J, Moayeri A et al - Isoflurane-Induced vasodilation on minimally increases cutaneous heat loss. Anesthesiology, 1991;74:226-232.

15. Sessler DI, Hynson J, Moayeri A et al - Thermoregulatory vasoconstriction decreases cutaneous heat loss. Anesthesiology, 1990;73:656-660.

16. Frank SM, Beattie C, Christopherson R et al - Unintentional hypothermia is associated with postoperative myocardial ischemia. The perioperative ischemia randomized anesthesia trial study group. Anesthesiology, 1993;78:468-476

17. Frank SM, Fleisher LA, Breslow MJ et al - Perioperative maintenance of normothermia reduces the incidence of morbid cardiac events. A randomized clinic trial. JAMA, 1997;277:1127-1134.

18. Morris RH - Operating room temperature and anesthetized, paralyzed patient. Arch Surg, 1971;102:95-97.

19. Kurz A, Sessler D, Narzt E et al - Postoperative hemodynamic and thermoregulatory consequences of intraoperative core hypothermia. J Clin Anesth, 1995;7:359-366.

20. Sharkey A, Lipton JM, Murphy MT et al - Inhibition of postanaesthetic shivering with radiant heat. Anesthesiology, 1987;66:249-252.

21. Imrie MM, Hall GM - Body temperature and anaesthesia. $\mathrm{Br} \mathrm{J}$ Anaesth, 1990;64:346-354.

22. Kurz A, Go JC, Sessler DI et al - Alfentanil slightly increases the sweating threshold and markedly reduces the vasoconstriction and shivering thresholds. Anesthesiology, 1995;83:293-299.

23. Kurz A, Ikeda T, Sessler DI et al - Meperidine decreases the shivering threshold twice as much as the vasoconstriction threshold. Anesthesiology, 1997;86:1046-1054.

24. Shehabi Y, Gatt S, Buckman T et al - Effect of adrenaline, fentanyl and warming of injectate on shivering following extradural analgesia in labour. Anaesth Intensive Care, 1990;18:31-37.

25. Cousins M, Cherry D, Gourlay G - Acute and Chronic Pain: Use of Spinal Opioids, em: Cousins M, Bridenbaugh P - Neural Blockade and Clinical Anesthesia and Pain Management. Philadelphia: JB Lippincott, 1988;955-1025.

26. Justins DM, Knott C, Lythman J et al - Epidural versus intramuscular fentanil. Analgesia and pharmacokinetics in labour. Anaesthesia. 1983;38:937-942.

\section{RESUMEN}

Abreu MP, Vieira JL, Lutti MN, Montarroyos ES, Rossi RT, Moraes R - Incidencia de Tremor en Anestesia Peridural Con y Sin Fentanil: Estudio Comparativo

JUSTIFICATIVA Y OBJETIVOS: La mayoría de los trabajos encontrados en la literatura, relacionando la influencia de los opioides administrados por vía peridural con el tremor per y pos-operatorio, fueron realizados con grupos de pacientes obstétricas, en las cuales la respuesta del centro termorregulador puede ser diferente de las pacientes no embarazadas. El objetivo de este trabajo fue comparar el bloqueo peridural con y sin fentanil, cuanto a la incidencia de tremores y otras complicaciones en el intra y pos-operatorio en pacientes sometidos a cirugía de várices bajo anestesia peridural con bupivacaína a 0,5\% con adrenalina a 1:200.000.

MÉTODO: Treinta y cuatro pacientes, estado físico ASA I y II, sometidos a cirugía para tratamiento de várices de miembros inferiores, fueron divididos aleatoriamente en 2 grupos $(n=17)$, y recibieron: midazolam $\left(0,05 \mathrm{mg} \cdot \mathrm{kg}^{-1}\right)$, por vía venosa seguido

Revista Brasileira de Anestesiologia Vol. 54, № 2, Março - Abril, 2004 
de anestesia peridural lumbar, utilizandose: en el grupo S, 20 $\mathrm{ml}$ bupivacaína a $0,5 \%$ (con vasoconstrictor) asociado a $2 \mathrm{ml}$ de solución fisiológica a 0,9\% y en el grupo $F, 20 \mathrm{ml} d e$ bupivacaína a $0,5 \%$ (con vasoconstrictor) asociada al fentanil $(100 \mu \mathrm{g})$. Fueron estudiados: incidencia de tremor, temperatura de los pacientes, necesidad del uso de meperidina, y la incidencia de náuseas y vómitos en los siguientes momentos: $M_{1}$ - admisión del paciente en la sala de operación; $M_{2}$ inmediatamente antes de la anestesia; $M_{3}-30$ minutos después del término de la inyección del anestésico local ( $A L)$; $M_{4}-60$ minutos después del término de la inyección de $A L ; M_{5}$ 90 minutos después del término de la inyección de $A L ; M_{6}$ - final de la anestesia; $M_{7}$ - antecediendo al alta de la sala de recuperación pos-anestésica.
RESULTADOS: Cuanto a los datos antropométricos, estado físico, tiempo medio de duración de la anestesia y cirugía, temperatura de los pacientes y de la sala de operación e incidencia de náuseas y vómitos no hubo diferente estadística entre los grupos. Hubo diferencia estadística a los 60 minutos (momento 4) y 90 minutos (momento 5) después del bloqueo peridural, con mayor incidencia de tremor en el Grupo-S que en el Grupo-F. Hubo mayor necesidad de utilización de meperidina en los pacientes sometidos al bloqueo peridural no asociado al fentanil.

CONCLUSIONES: En las condiciones de este estudio, la adición de $100 \mu \mathrm{g}$ de fentanil al anestésico local, por vía peridural, mostró que el opioide no tiene la propiedad de suprimir el tremor, más de reducir su incidencia y la intensidad, sin aumentar la incidencia de náuseas y vómitos. 